\title{
How an Active Liquid Wets a Surface
}

\author{
The conditions that make liquid stick to a permeable membrane could \\ elucidate how bacteria form films, and help researchers design tiny \\ swimming robots.
}

\section{By Sophia Chen}

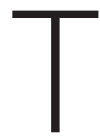

he study of active particles suspended in mixtures of liquid and gas has applications ranging from biology to robotics, where insights could explain how bacteria form films on surfaces and help researchers design self-propelled microrobots. In these settings, understanding the process of wetting-how a liquid makes contact with a surface-is crucial. In new theoretical work, Francesco Turci and Nigel Wilding of the University of Bristol, UK, have identified conditions that influence wetting interactions in an active fluid, finding unexpected parallels with the wetting behavior of conventional liquids [1].

The researchers study 2D and 3D versions of a model in which active Brownian particles-self-propelled particles that move in random directions-interact with a permeable barrier. Forces between the particles are purely repulsive, and the barrier also repels the particles with a strength that Turci and Wilding tune.

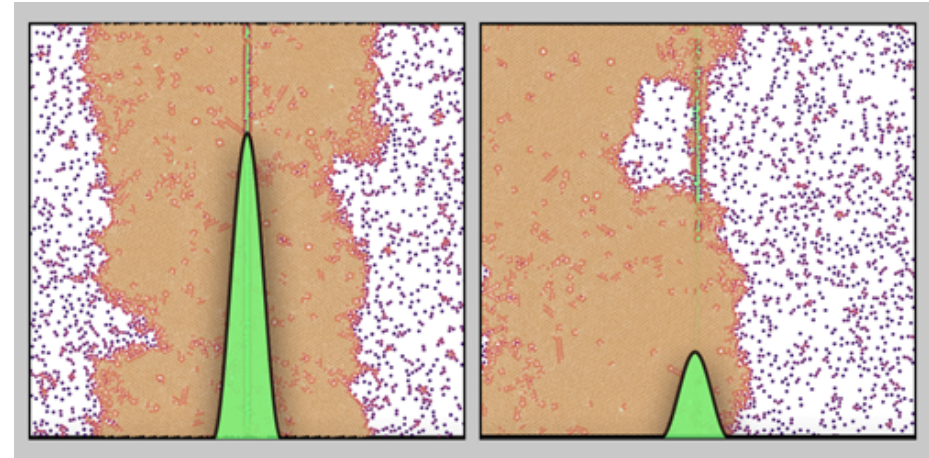

Credit: Adapted from F. Turci and N. B. Wilding [1]
The researchers found that, despite including only repulsive interactions, their model reproduced wetting behaviors that, in conventional liquids, are governed by attractive forces. When the barrier repelled the particles strongly, the active liquid accumulated on both sides of the barrier in a configuration that the researchers liken to a surface fully wetted by a conventional liquid. When the barrier's repulsion was weak, the active liquid stayed on one side of the barrier without making contact-an arrangement resembling a fully dry surface. Intermediate repulsive strengths caused the barrier to be partially wetted. Also paralleling the behavior of a conventional liquid, the transition to a partially wetted state occurred gradually in the 2D model and abruptly in the 3D model. In future work, the researchers plan to verify their findings experimentally using active matter such as robot swarms.

Sophia Chen is a freelance science writer based in Columbus, Ohio.

\section{REFERENCES}

1. F. Turci and N. B. Wilding, "Wetting transition of active Brownian particles on a thin membrane," Phys. Rev. Lett. 127, 238002 (2021). 